\title{
Comparison of prognosis for men with type 2 diabetes mellitus and men with cardiovascular disease
}

\author{
Gilles R. Dagenais MD, Annie St-Pierre PhD, Patrick Gilbert MD, Benoît Lamarche PhD, \\ Jean-Pierre Després PhD, Paul-Marie Bernard MSc, Peter Bogaty MD
}

\section{ABSTRACT}

Background: People with type 2 diabetes mellitus are at high risk for cardiovascular disease. In some studies, the mortality rate among people with this condition has been equivalent to that among people with cardiovascular disease. We compared cardiovascular mortality between incident cases of diabetes and cardiovascular disease.

Methods: The study population was part of a random sample of 4376 men from Quebec, Canada, aged 35 to 64 years, who did not have cardiovascular disease in 1974 and who were followed until 1998. Three groups of incident cases were identified: diabetes without cardiovascular disease, first cardiovascular event (myocardial infarction, unstable angina or stroke) without diabetes, and both cardiovascular disease and diabetes. These cases were age-matched to a control group without diabetes or cardiovascular disease.

Results: During the 24-year follow-up period, new diabetes without cardiovascular disease was documented in 137 men. A first cardiovascular event without diabetes was documented in 527 men. Relative to the 627 controls, men with 1 of the 2 diseases of interest had higher cardiovascular mortality (age-adjusted relative risk [RR] 3.11, 95\% confidence interval $[\mathrm{Cl}]$ 1.96-4.92) for those with diabetes and 4.46 (95\% Cl 3.15-6.30) for those with cardiovascular disease). However, within the first 5 years after diagnosis, men with cardiovascular disease had higher cardiovascular mortality than men with diabetes (age-adjusted RR 2.03, 95\% Cl 1.01-4.08).

Interpretation: Men with isolated type 2 diabetes and men with isolated cardiovascular disease had similar cardiovascular mortality rates several years after initial diagnosis of either condition. These findings reinforce the need to prevent and optimally manage diabetes and cardiovascular disease.

Une version française de ce résumé est disponible à l'adresse www.cmaj.ca/cgi/content/full/180/1/40/DC1

CMAJ 2009;180(1):40-7

厂 n 1971, type 2 diabetes mellitus was already considered an epidemic, affecting more than 170 million people worldwide. ${ }^{1}$ In 2001, it was estimated that diabetes prevalence would increase by nearly $50 \%$ by the year 2010 . Epidemiologic studies performed in randomly sampled populations and initiated in the 1970s and 1980s have shown that diabetes increases the risk of all-cause death, as well as death due to cardiovascular disease and coronary artery disease. ${ }^{2-23}$ In some studies, ${ }^{12-17}$ but not all studies, ${ }^{18-23}$ coronary or cardiovascular mortality among people with type 2 diabetes without previous cardiovascular disease was equivalent to that of people without diabetes who had had a first myocardial infarction or first cardiovascular event. Thus, there is controversy as to whether diabetes alone confers a risk of cardiovascular mortality similar to that associated with having had a first coronary or cardiovascular event. The differences in findings among various studies may be attributable to several factors such as age, sex, duration of diabetes and cardiovascular disease, ethnicity, cardiovascular risk factors and therapies. Furthermore, most studies used prevalent cases without considering the duration of cardiovascular disease or diabetes and did not exclude highrisk patients who had angina or intermittent claudication before the diagnoses of diabetes and cardiovascular disease.

The rationale for undertaking the present study was the need for more information about the cardiovascular prognosis of men with type 2 diabetes relative to men with cardiovascular disease. We used incident instead of prevalent cases, without prior angina and without intermittent claudication. We speculated that the adverse prognosis associated with a diagnosis of diabetes would be similar to the prognosis associated with a diagnosis of cardiovascular disease over the long term but would be less similar over the short term. We formulated 2 hypotheses: first, that within the first few years after diagnosis, the risk of a fatal cardiovascular event would be higher among men with a first cardiovascular event and no diabetes than among men with type 2 diabetes and no cardiovascular disease; and second, that over the longer term, the risk of death within these 2 clinical subsets would tend toward equivalence.

\section{Methods}

\section{Study design and participants}

The study population was part of the Quebec Cardiovascular Study, a prospective cohort study, which has been de-

From the Institut universitaire de cardiologie et pneumologie (Dagenais, St-Pierre, Després, Bogaty), the Institut de nutraceutiques et aliments fonctionnels (Lamarche) and the Faculté de médicine (Bernard), Université Laval, Québec, Que.; and the Centre Hospitalier de I'Université de Montréal (Gilbert), Montréal, Que. 


\section{Box 1: Definitions of incident cases and outcomes}

- Type 2 diabetes was either self-reported or diagnosed on the basis of fasting plasma glucose $\geq 7.0 \mathrm{mmol} / \mathrm{L}$.

- Myocardial infarction required at least 2 of the following 3 features: characteristic symptoms, electrocardiogram showing new Q-wave or evolving ST-segment elevation or cardiac enzymes at least twice the upper limit of the normal range.

- Unstable angina required typical symptoms and an electrocardiographic tracing showing characteristic ischemic ST-T changes.

- Stroke required a sudden neurological deficit of vascular origin, lasting at least 24 hours, but excluding trauma, tumour or malformation.

scribed previously. ${ }^{24,25}$ Briefly, from December 1973 to June 1974, we asked a large sample of men who were 35 to 64 years of age in 1973 and who represented $65 \%$ of a random sample of the suburbs in the city of Québec, Canada (according to the age strata of men in the province of Quebec) to participate first in an assessment of risk factors and then in a prospective evaluation. We excluded men with clinical evidence of cardiovascular disease (as indicated by a medical examination and electrocardiographic findings on entry to the study). For the present report, we also excluded men who had diabetes before study entry and those who had effort angina or intermittent claudication before diagnosis of diabetes or diagnosis of first myocardial infarction, unstable angina or stroke.

The study was approved by the Comité d'éthique de la recherche de la Faculté de medicine de l'Université de Montréal. Participants gave written consent for data to be gathered from their medical charts.

\section{Data collection}

At study entry, each man answered a standardized validated medical questionnaire administered by a specially trained nurse and underwent a medical examination, which included electrocardiography. Smoking was self-reported, and men were classified as smokers or nonsmokers (defined as never smoked or discontinued smoking a year or more before entry into the study). We computed baseline blood pressure as the mean of 2 blood pressure measurements taken 5 minutes apart with a standard mercury sphygmomanometer after the participant had been seated for at least 5 minutes. Each man provided a nonfasting blood sample for determination of serum cholesterol. We defined level of education as number of years of attendance at school. For each participant, we documented family history of coronary artery disease and stroke (father, mother, brother or sister). We determined baseline body mass index (BMI) for participants for whom we had the necessary data for this calculation.

Participants returned to the hospital clinic for reevaluation in 1980 and 1985. At these follow-up visits, each man answered a standardized and validated medical ques- tionnaire, underwent blood pressure measurements and electrocardiography, and provided fasting blood samples for the determination of lipid profile in the fasting state. At the 1985 evaluation, several participants were not fasting when they arrived at the clinic, so we could not obtain their fasting lipid profiles. Participants who were unable to attend the clinic in person (because they had moved from the city of Québec, lived in a retirement home or were in hospital) answered the questionnaire by telephone or by mail. These participants did not undergo a medical examination, but we obtained information on their health status and any hospital stays. In 1990 and 1997-1998, the men answered a standardized questionnaire about their medical status by letter or telephone.

Men with incident cases of diabetes or cardiovascular disease occurring between 1974 and 1998 were divided into 3 groups: those with incident type 2 diabetes but without cardiovascular disease; those with a first myocardial infarction, first episode of unstable angina or first stroke without diabetes or previous cardiovascular event; and those with both type 2 diabetes and a first myocardial infarction, first episode of unstable angina or first stroke without other previous cardiovascular event. Clinical definitions appear in Box 1. The control group consisted of men without diabetes or cardiovascular disease, age-matched to men in the 3 other groups. Both incident cases and controls were followed from the incidence (sampling) time to the events or the end of study.

\section{Outcomes}

The main outcome was fatal cardiovascular disease, including death due to coronary artery disease, stroke, congestive heart failure, pulmonary embolism or ruptured aneurysm, as well as sudden death or cardiovascular periprocedural death. The other outcome was total mortality. Three cardiologists used hospital charts to adjudicate incident cases and outcomes. For deaths that occurred outside the hospital, we contacted the patient's next-of-kin to document the circumstances of death. We confirmed this information by checking the death certificate or a note from the provincial registry or the physician who attested the death.

\section{Statistical analysis}

We performed univariable and age-adjusted analyses using Cox regression models. We applied age-stratified modelling (age dichotomized as $<60$ years or $\geq 60$ years old). Relative risks (RRs) are presented with their $95 \%$ confidence intervals (CIs). We computed Kaplan-Meier survival probability in each group for cardiovascular mortality and total mortality. We used the log-rank test to compare survival curves among groups. We did not adjust for systolic blood pressure, smoking status, serum cholesterol, family history of cardiovascular disease or any other factors except age, because for most patients these risk factors were unknown at the incidence time or during follow-up. Nevertheless, we characterized the risk factor status of the 4 groups at baseline (in 1974) and in 1985 and compared them using analysis of variance $F$ tests or $\chi^{2}$ tests. 


\section{Results}

\section{Study population}

A total of 4637 men initially agreed to participate in the Quebec Cardiovascular Study. We excluded 245 men at the time of study entry, because they already had clinical evidence of cardiovascular disease. An additional 16 men were lost to follow-up after the first evaluation, which left a sample of 4376 participants. Follow-up was incomplete for a further 155 (3.5\%) men: we determined that 31 men were still living in 1977 but were subsequently lost to follow-up, 23 were still living in 1979 but were subsequently lost to follow-up, 3 were still living in 1981 but were subsequently lost to follow-up, and 98 were still living in 1990 but were subsequently lost to follow-up. Available data for these participants were included in the study.

In $1974,3066(70.1 \%)$ of the 4376 men were smokers, and 854 (19.5\%) had blood pressure above 160/95 mm Hg; these 2 characteristics were inversely related to the number of years at school. A total of 426 (9.7\%) men had serum cholesterol of at least $6.2 \mathrm{mmol} / \mathrm{L}$, but this characteristic was not inversely related to the number of years at school. At baseline, we determined BMI for 2108 (48.2\%) of the 4376 participants.

Table 1: Characteristics of men according to diagnosis of diabetes and cardiovascular disease (CVD) and outcome

\begin{tabular}{|c|c|c|c|c|c|c|}
\hline \multirow[b]{2}{*}{ Characteristic } & \multicolumn{4}{|c|}{ Group; mean (SD)* } & \multicolumn{2}{|c|}{$p$ value } \\
\hline & $\begin{array}{c}\text { Neither diabetes } \\
\text { nor CVD }\end{array}$ & $\begin{array}{c}\text { Diabetes and } \\
\text { no CVD }\end{array}$ & $\begin{array}{l}\text { CVD and no } \\
\text { diabetes }\end{array}$ & $\begin{array}{l}\text { CVD and } \\
\text { diabetes }\end{array}$ & $\begin{array}{c}\text { Group } \\
\text { differences }\end{array}$ & $\begin{array}{c}\text { Diabetes and no } \\
\text { CVD v. CVD and } \\
\text { no diabetes }\end{array}$ \\
\hline Baseline (1974) & $n=627$ & $n=137$ & $n=527$ & $n=18$ & & \\
\hline Age, yr & $48.5(7.8)$ & $48.9(7.7)$ & $49.0(8.0)$ & $52.1(6.2)$ & 0.21 & 0.91 \\
\hline Systolic blood pressure, $\mathrm{mm} \mathrm{Hg}$ & $137.1(18.0)$ & $146.2(19.4)$ & $143.1(20.0)$ & $152.9(24.5)$ & $<0.001$ & 0.10 \\
\hline Diastolic blood pressure, $\mathrm{mm} \mathrm{Hg}$ & $83.8(10.3)$ & $89.6(11.1)$ & $87.0(11.3)$ & $92.1(14.4)$ & $<0.001$ & 0.016 \\
\hline $\mathrm{BMI},+\mathrm{kg} / \mathrm{m}^{2}$ & $25.4 \quad(3.7)$ & $28.8(4.7)$ & $25.8(3.9)$ & $29.7(6.1)$ & $<0.001$ & $<0.001$ \\
\hline Cholesterol, $\mathrm{mmol} / \mathrm{L}$ & $4.81(0.90)$ & $4.92(1.00)$ & $5.11(1.10)$ & $5.40(0.80)$ & $<0.001$ & 0.051 \\
\hline Years at school & 9.8 (3.5) & 9.4 (3.9) & $10.0(3.8)$ & $8.1 \quad(3.0)$ & 0.07 & 0.11 \\
\hline Family history of CVD, no. (\%) & $150(23.9)$ & $42 \quad(30.7)$ & $163(30.9)$ & $2(11.1)$ & 0.008 & 0.85 \\
\hline Current smoker, no. (\%) & $439(70.0)$ & $101(73.7)$ & $400(75.9)$ & $12(66.7)$ & 0.18 & 0.60 \\
\hline In 1985‡ & $n=598$ & $n=125$ & $n=488$ & $n=17$ & & \\
\hline Age, yr & $59.1(7.6)$ & $59.3(7.3)$ & $59.4(7.8)$ & $62.5(5.8)$ & 0.30 & 0.91 \\
\hline Systolic blood pressure, $\S \mathrm{mm} \mathrm{Hg}$ & $133.2(18.6)$ & $138.1(18.9)$ & $136.7(19.3)$ & $139.7(19.4)$ & 0.010 & 0.50 \\
\hline Diastolic blood pressure, $\S \mathrm{mm} \mathrm{Hg}$ & $81.4(10.8)$ & $81.1(9.7)$ & $82.3(11.3)$ & $81.0(11.4)$ & 0.56 & 0.32 \\
\hline $\mathrm{BMI},+\mathrm{kg} / \mathrm{m}^{2}$ & $26.0(3.6)$ & $28.7(3.9)$ & $26.5(4.3)$ & $28.1(4.2)$ & $<0.001$ & $<0.001$ \\
\hline Cholesterol, $\rceil \mathrm{mmol} / \mathrm{L}$ & $5.67(1.00)$ & $5.52(1.00)$ & $5.93(1.20)$ & $5.96(1.00)$ & 0.004 & 0.008 \\
\hline HDL cholesterol, ** $\mathrm{mmol} / \mathrm{L}$ & $1.06(0.27)$ & $0.93(0.23)$ & $0.97(0.24)$ & $0.96(0.21)$ & $<0.001$ & 0.13 \\
\hline LDL cholesterol, †† mmol/L & $3.82(0.90)$ & $3.58(0.80)$ & $4.05(0.99)$ & $3.94(1.10)$ & $<0.001$ & $<0.001$ \\
\hline Apolipoprotein B, §§ mg/dL & $114.9(30.4)$ & $117.6(29.9)$ & $126.1(33.9)$ & $124.0(28.9)$ & $<0.001$ & 0.053 \\
\hline Family history of CVD, \% & $224(37.5)$ & $55(44.0)$ & $260(53.3)$ & $11(64.7)$ & $<0.001$ & 0.06 \\
\hline Current smoker, \% & $198(33.1)$ & $37(29.6)$ & $196(40.2)$ & $9(52.9)$ & 0.017 & 0.026 \\
\hline Outcome & $n=627$ & $n=137$ & $n=527$ & $n=18$ & & \\
\hline Total deaths, no. (\%) & $140(22.3)$ & $60(43.8)$ & $204(38.7)$ & $9(50.0)$ & & \\
\hline Total CVD deaths, no. (\%) & $43(6.9)$ & $32(23.4)$ & $129(24.5)$ & 7 (38.9) & & \\
\hline
\end{tabular}

Note: $\mathrm{BMI}=$ body mass index, $\mathrm{HDL}=$ high-density lipoprotein, $\mathrm{LDL}=$ low-density lipoprotein, $\mathrm{SD}=$ standard deviation.

*Unless indicated otherwise.

tIn 1974, body mass index was measured in $45 \%$ of men with neither diabetes nor cardiovascular disease, in $53 \%$ of those with diabetes only, in $56 \%$ of those with cardiovascular disease only, and in $45 \%$ of those with both conditions. In 1985, BMI was measured in $72 \%$ of men with neither diabetes nor cardiovascular disease, in $62 \%$ of those with diabetes only, in $83 \%$ of men with cardiovascular disease, and $94 \%$ of those with both conditions.

$\ddagger$ Numbers of participants in each group were lower in 1985 because of deaths since baseline.

§In 1985 , blood pressure was measured in $82 \%$ of men with neither diabetes nor cardiovascular disease, in $80 \%$ of those with diabetes only, in $83 \%$ of those with cardiovascular disease only, and in $94 \%$ of those with both conditions.

IIn 1985, total cholesterol was measured in $59 \%$ of men with neither diabetes nor cardiovascular disease, in $50 \%$ of those with diabetes only, in $57 \%$ of those with cardiovascular disease only, and in $41 \%$ of those with both conditions.

**In 1985, HDL cholesterol was measured in $59 \%$ of men with neither diabetes nor cardiovascular disease, in $54 \%$ of those with diabetes only, in $57 \%$ of those with cardiovascular disease only, and in $41 \%$ of those with both conditions.

t+In 1985, LDL cholesterol was measured in $58 \%$ of men with neither diabetes nor cardiovascular disease, in $52 \%$ of those with diabetes only, in $56 \%$ of those with cardiovascular disease only, and in $41 \%$ of those with both conditions.

$\S \S$ In 1985, apolipoprotein B was measured in $59 \%$ of men with neither diabetes nor cardiovascular disease, in $54 \%$ of those with diabetes only, in $57 \%$ of those with cardiovascular disease only, and in $41 \%$ of those with both conditions. 
Between 1974 and 1985, 1112 men died. For the remaining 3264 men, information was complete for age and smoking status. We obtained blood pressure measurements for $2676(82.0 \%)$ of 3264 participants and the lipid profile (total cholesterol, low-density lipoprotein [LDL] cholesterol, highdensity lipoprotein [HDL] cholesterol and apolipoprotein B) for $1828(56.0 \%)$ of these 3264 participants.

During the 24-year follow-up, 137 men had a new diagnosis of type 2 diabetes without any previous cardiovascular disease, 527 men had a first nonfatal cardiovascular event (myocardial infarction in 354, unstable angina in 58 and stroke in 115) without diabetes, and 18 had a new diagnosis of a cardiovascular event (myocardial infarction in 12, unstable angina in 1 and stroke in 5) and diabetes. We agematched 627 of these 682 incident cases to control participants (Table 1). At study entry, men who subsequently had diabetes without cardiovascular disease had higher diastolic blood pressure and greater BMI than men who subsequently had a first cardiovascular event without diabetes, but there was no significant difference in age, serum cholesterol, smoking status or family history of cardiovascular disease between these 2 groups. As expected, men with neither diabetes nor cardiovascular disease had lower blood pressure and serum cholesterol, and a lower proportion of these men were smokers.

For each participant, we documented changes in risk factors between 1974 and 1985. The most important changes over this period were smoking status, blood pressure and serum cholesterol level. The rate of smokers decreased from $73.7 \%$ to $29.6 \%$ in the group of men with incident diabetes but no cardiovascular disease, from $75.9 \%$ to $40.2 \%$ in the group with incident cardiovascular disease but no diabetes, from $67.0 \%$ to $52.9 \%$ in the group with both incident cardiovascular disease and incident diabetes, and from $70.0 \%$ to $33.1 \%$ in the control group (Table 1). Relative to the time of entry into the study (in 1974), systolic and diastolic blood pressures were lower in all groups in 1985, and there was no difference between men with incident diabetes and men with incident cardiovascular disease. Serum cholesterol increased in each group; this may be attributable to a change in measurement method. Men with incident cardiovascular disease and no diabetes had a higher serum cholesterol level than men with incident diabetes and no cardiovascular disease. Although only $56 \%$ of the cohort had a complete lipid profile in 1985, participants with cardiovascular disease alone and cardiovascular disease in combination with diabetes had the highest levels of LDL cholesterol and apolipoprotein B, and a greater proportion of these groups were smokers. Relative to men with incident cardiovascular disease and no diabetes, those with incident diabetes and no cardiovascular disease had greater BMI.

The mean age of participants was 55.2 (standard deviation [SD] 9.0) years at the time of diagnosis of diabetes among those with incident diabetes, 61.0 (SD 9.3) years at the time of an acute cardiovascular event among those with incident cardiovascular disease, and 58.8 (SD 9.0) years for the control

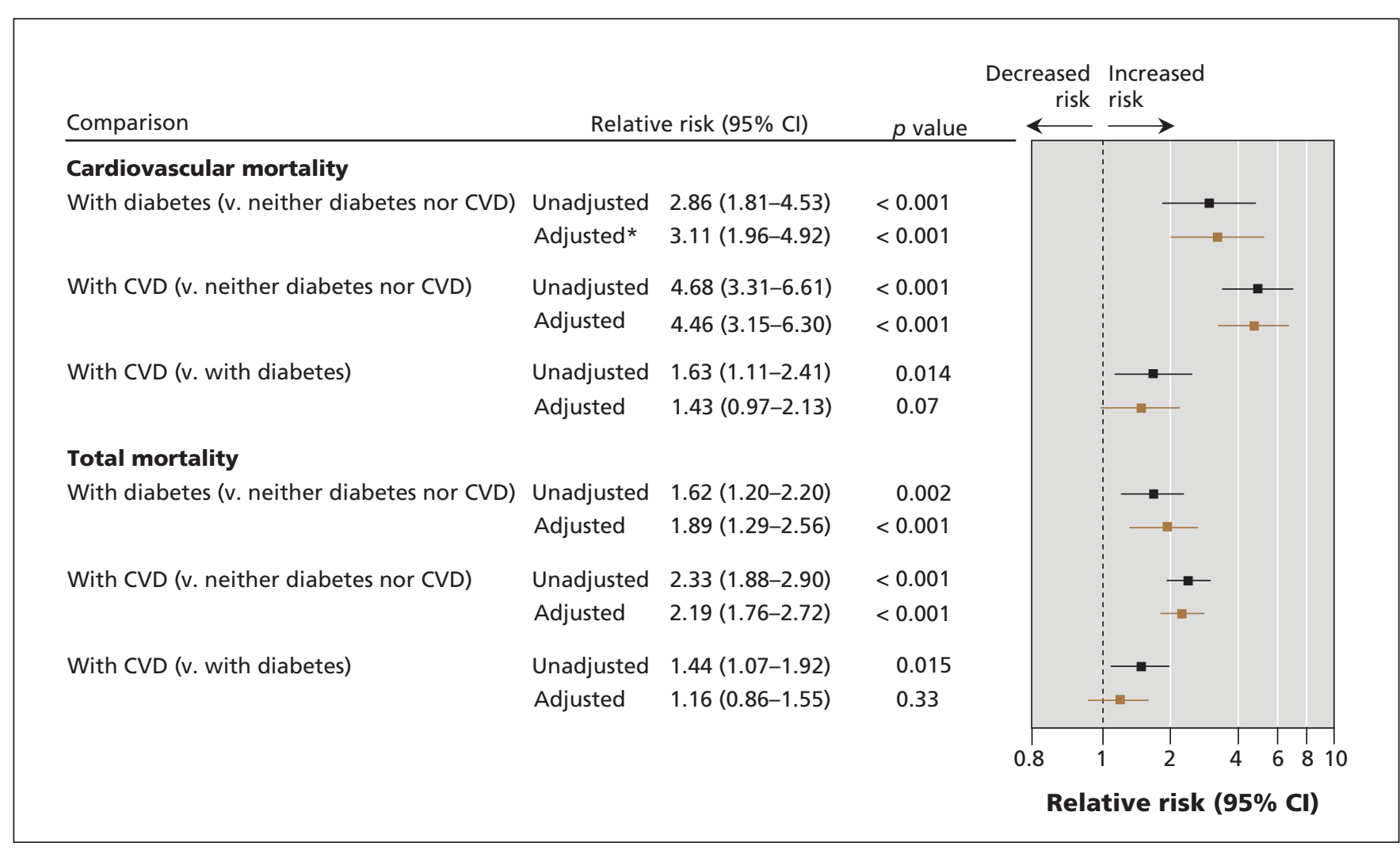

Figure 1: Cardiovascular and total mortality among men with diabetes or cardiovascular disease (CVD). *Adjusted for age (dichotomized as $<60 \mathrm{yr}$ and $\geq 60 \mathrm{yr}$ ). Note: $\mathrm{Cl}=$ confidence interval. 
group at the time of selection for matching with the incident cases ( $p<0.001$ among the 3 groups). At entry into the study in $1973-1974,171(3.9 \%)$ of the 4376 participants were taking antihypertensive agents, mostly diuretics. Of the 3264 participants in 1985, only $186(5.7 \%)$ were taking $\beta$-blockers, 95 $(2.9 \%)$ were taking diuretics and $62(1.9 \%)$ were taking lipidlowering agents (clofibrate and cholestyramine). From 1985 to 1998, documentation of therapy was obtained for only 2646 participants. Diuretics had been prescribed for 299 men (11.3\%), $\beta$-blockers for 243 (9.2\%), calcium channel blockers for $180(6.8 \%)$, angiotensin-converting enzyme inhibitors for $29(1.1 \%)$ and lipid-lowering agents for $127(4.8 \%)$.

\section{Outcomes}

Cardiovascular death was documented in $23.4 \%$ of men with incident diabetes and no cardiovascular disease, and $6.9 \%$ of

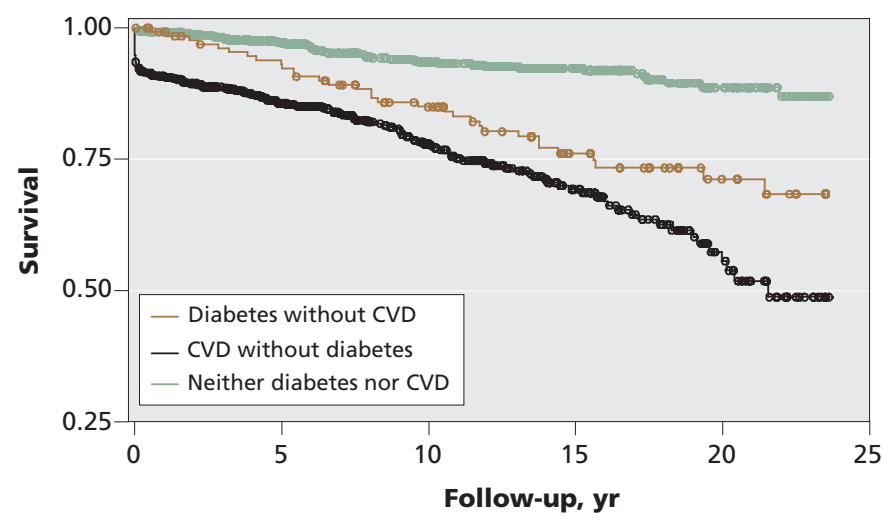

Figure 2: Unadjusted Kaplan-Meier survival curves for cardiovascular disease mortality among men with neither diabetes nor cardiovascular disease, men with diabetes and no cardiovascular disease, and men with a first acute cardiovascular event and no diabetes. Log-rank test: $p<0.001$.

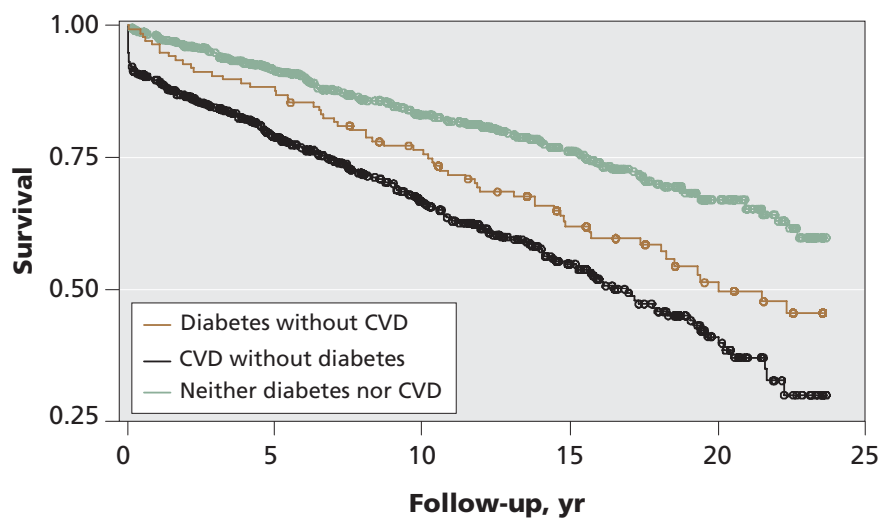

Figure 3: Unadjusted Kaplan-Meier survival curves for all-cause mortality among men with neither diabetes nor cardiovascular disease, men with diabetes and no cardiovascular disease, and men with a first acute cardiovascular event and no diabetes. Log-rank test: $p<0.001$. controls (Table 1). Death from any cause was documented in $43.8 \%$ of men with diabetes and no cardiovascular disease and $22.3 \%$ of controls. Compared with the control group, the age-adjusted RR of cardiovascular mortality among men with incident diabetes and no cardiovascular disease was 3.11 (95\% CI 1.96-4.92), and the age-adjusted RR of total mortality was 1.89 (95\% CI 1.29-2.56) (Figure 1).

Among men with incident cardiovascular disease and no diabetes, cardiovascular death was documented in $24.5 \%$, and death from any cause was documented in $38.7 \%$ (Table 1). The age-adjusted RR in this group, relative to controls, was 4.46 (95\% CI 3.15-6.30) for cardiovascular mortality and 2.19 (95\% CI 1.76-2.72) for all-cause mortality (Figure 1).

Among men with both diabetes and cardiovascular disease, cardiovascular death was documented in $38.9 \%$, and death from any cause was documented in $50.0 \%$. The number of men in this category was only 18 , so we did not include this group in the analyses presented in Figure 1 or in the survival curves.

Unadjusted survival rates were highest for controls, followed by men with incident diabetes but without cardiovascular disease and then by men with incident cardiovascular disease but without diabetes (Figure 2) $(p<0.001)$. Men with cardiovascular disease alone had lower survival early in the follow-up period. However, from the 5th to the 10th year, the survival curve for this group ran parallel to the curve for men with diabetes and no cardiovascular disease. The mean period between diagnosis of diabetes and a fatal cardiovascular event was 8.7 (SD 5.4) years. The mean period between diagnosis of cardiovascular disease and cardiovascular death was 6.0 (SD 6.4) years. Compared with men with incident diabetes, those with incident cardiovascular disease had higher cardiovascular mortality, but this higher rate did not reach statistical significance when adjusted for age (RR 1.43, 95\% CI 0.97-2.13). A similar pattern was observed for total mortality (RR 1.16, 95\% CI 0.86-1.55) (Figure 1, Figure 3). However, the age-adjusted risk for cardiovascular mortality within the first 5 years after diagnosis was twice as high for men with incident cardiovascular disease as for men with incident diabetes (age-adjusted RR 2.03, 95\% CI 1.01-4.08). Thereafter, there was no significant difference between these 2 groups (age-adjusted RR 1.15, 95\% CI 0.70-1.88) (Table 2).

\section{Interpretation}

In this study, we sought to determine whether men with isolated type 2 diabetes had a prognosis equivalent to that of men with isolated cardiovascular disease. We used a different methodologic approach than what has been used in previous studies. Our approach was based on incident, rather than prevalent, cases of diabetes and cardiovascular disease without previous angina or intermittent claudication, both of which are known to increase the risk of cardiovascular disease.

Our first hypothesis was that men with type 2 diabetes but no previous cardiovascular disease would have a risk for fatal cardiovascular events lower than that for men with a first myocardial infarction, unstable angina or stroke without diabetes during the first few years after initial diagnosis of diabetes or the acute cardiovascular event. Our second hy- 
Table 2: Cardiovascular and total mortality among men with diabetes or cardiovascular disease (CVD) according to duration of follow-up

\begin{tabular}{|c|c|c|c|c|}
\hline \multirow[b]{2}{*}{ Comparison } & \multicolumn{2}{|c|}{ Follow-up $<5$ years } & \multicolumn{2}{|c|}{ Follow-up $\geq 5$ years } \\
\hline & Relative risk $(95 \% \mathrm{Cl})$ & $p$ value & Relative risk $(95 \% \mathrm{Cl})$ & $p$ value \\
\hline \multicolumn{5}{|l|}{ Cardiovascular mortality } \\
\hline \multicolumn{5}{|l|}{ Unadjusted } \\
\hline With diabetes v. neither diabetes nor CVD & $2.51(1.10-5.68)$ & 0.027 & $2.98(1.71-5.20)$ & $<0.001$ \\
\hline With CVD v. neither diabetes nor CVD & $5.76(3.35-9.91)$ & $<0.001$ & $3.92(2.48-6.20)$ & $<0.001$ \\
\hline With CVD v. with diabetes & $2.30(1.15-4.59)$ & 0.019 & $1.32(0.81-2.14)$ & 0.27 \\
\hline \multicolumn{5}{|l|}{ Adjusted for age at diagnosis* } \\
\hline With diabetes v. neither diabetes nor CVD & $2.72(1.20-6.17)$ & 0.016 & $3.24(1.85-5.67)$ & $<0.001$ \\
\hline With CVD v. neither diabetes nor CVD & $5.52(3.21-9.50)$ & $<0.001$ & $3.72(2.35-5.89)$ & $<0.001$ \\
\hline With CVD v. with diabetes & $2.03(1.01-4.08)$ & 0.047 & $1.15(0.70-1.88)$ & 0.58 \\
\hline \multicolumn{5}{|l|}{ Total mortality } \\
\hline \multicolumn{5}{|l|}{ Unadjusted } \\
\hline With diabetes v. neither diabetes nor CVD & $1.51(0.87-2.62)$ & 0.14 & $1.66(1.15-2.38)$ & 0.007 \\
\hline With CVD v. neither diabetes nor CVD & $2.77(1.98-3.88)$ & $<0.001$ & $2.04(1.53-2.71)$ & $<0.001$ \\
\hline With CVD v. with diabetes & $1.84(1.10-3.07)$ & 0.020 & $1.23(0.86-1.76)$ & 0.26 \\
\hline \multicolumn{5}{|l|}{ Adjusted for age at diagnosis* } \\
\hline With diabetes v. neither diabetes nor CVD & $1.77(1.02-3.08)$ & 0.043 & $1.92(1.33-2.77)$ & $<0.001$ \\
\hline With CVD v. neither diabetes nor CVD & $2.55(1.82-3.58)$ & $<0.001$ & $1.93(1.45-2.58)$ & $<0.001$ \\
\hline With CVD v. with diabetes & $1.44(0.86-2.42)$ & 0.17 & $1.01(0.70-1.45)$ & 0.96 \\
\hline
\end{tabular}

Note: $\mathrm{Cl}=$ confidence interval.

*Age dichotomized as $<60 \mathrm{yr}$ and $\geq 60 \mathrm{yr}$.

pothesis was that, over the longer term, the risk of fatal cardiovascular events for these subsets of participants would tend to become similar. Both hypotheses were confirmed by the study results. Compared with men with incident cardiovascular disease, men with incident diabetes had a lower risk for cardiovascular mortality during the first 5 years after their diagnosis of diabetes, but subsequently there was no statistically significant difference between the 2 groups for cardiovascular or total mortality. Our study also highlighted the high risk of death associated with diabetes: for men with incident type 2 diabetes and no previous cardiovascular disease, the risk of cardiovascular mortality was 3 times greater than for men with neither cardiovascular disease nor diabetes at the beginning of follow-up.

As expected, cardiovascular mortality was higher during the first 5 years of follow-up for men with a first acute cardiovascular event than for men with incident diabetes and no cardiovascular disease. The similar long-term (beyond 5 years) cardiovascular risk for participants with incident diabetes or cardiovascular disease has been previously reported in some ${ }^{12-17}$ but not all studies, specifically for men. ${ }^{16-23}$

The discrepancy between our results and those of previous studies may be attributable to several other variables in addition to the methodologic approach. Longer duration of diabetes is associated with an increased risk of cardiovascular disease. ${ }^{3,19}$ In our study, men with incident diabetes and no cardiovascular disease were at greater risk when they were known to have had diabetes for more than 5 years. A longer duration of diabetes likely aggravates the atherothrombotic process that is associated with diabetes. ${ }^{3}$ Another important prognostic factor was the age of participants. Booth and colleagues ${ }^{13}$ reported that for men in Ontario with diabetes and no myocardial infarction, the rate of the composite of allcause death and acute myocardial infarction was similar to that for men with recent acute myocardial infarction and no diabetes during a 6-year follow-up only if they were at least 50 years of age. Most men with diabetes in our study were older than this age. In other studies, women with diabetes were at greater risk than men with diabetes, and their risk of cardiovascular mortality was similar to that of women with cardiovascular disease..$^{15-17}$

In most studies, researchers have adjusted survival for baseline risk factors, but only a few researchers have made these adjustments during long-term follow-up. We did not adjust for risk factors other than age because we did not have the necessary data for risk factor evaluation for the incident cases over the 24-year follow-up period. In our study, there were major changes in the risk factors between 1974 and 1985. Smoking rates declined from $73.7 \%$ to $29.6 \%$ and blood pressure declined from $146 / 90$ to $138 / 81 \mathrm{~mm} \mathrm{Hg}$ among men with incident diabetes and no cardiovascular disease, and smoking rates declined from $75.9 \%$ to $40.2 \%$ and blood pressure from $143 / 87$ to $137 / 82 \mathrm{~mm} \mathrm{Hg}$ among men with incident cardiovascular disease and no diabetes. In 1985, men with cardiovascular disease 
were at greater risk for cardiovascular death than men with diabetes, considering their rate of smoking and lipid profile.

Contrary to our study, other studies have not excluded participants with angina or intermittent claudication or adjusted for these conditions. In several studies, participants selfreported events, and researchers did not adjudicate death but rather obtained the data from registries. In our study, we adjudicated all events. Thus, we believe that the duration of diabetes and the age of men with this condition were the main factors contributing to the high risk among men with incident diabetes and no cardiovascular disease.

\section{Limitations}

Our study had limitations. First, it was limited to men, all of whom happened to be white. Second, diabetes was selfreported in two-thirds of cases. This approach is considered reliable for those who are aware of their illness ${ }^{26}$ or who have a fasting blood glucose level of at least $7.0 \mathrm{mmol} / \mathrm{L}$, but we may have missed men with diabetes who did not have a blood glucose determination, and we may have misclassified some men with diabetes. Such misclassification should have attenuated the association between diabetes and cardiovascular events, resulting in an underestimation of the risk in the diabetic cohort. Underestimation was also most likely present in the group with incident cardiovascular disease because that group was selected according to rigorously defined criteria, ignoring, for example, unstable angina without clearly ischemic electrocardiographic changes or myocardial infarction with cardiac enzymes that were not above twice the upper limit of normal (Box 1). In our study, we focused on men with diabetes and men without diabetes; however, within the group of men without diabetes, some participants might have had impaired fasting glucose or glucose intolerance, which are known to increase the risk of cardiovascular events. ${ }^{8,11,27}$

We did not adjust for other confounding variables except age during the course of the follow-up, but we excluded other cardiovascular disease before the diagnosis of acute cardiovascular disease or diabetes. Except for the Framingham Heart Study, other long-term studies have adjusted for other variables only at baseline or, in some studies, during the first years after entry in the study but not during most of the longterm follow-up.

Pharmacologic interventions such as statins, antiplatelet agents, modulators of the renin angiotensin system and $\beta$ blockers have been shown to reduce cardiovascular disease in patients who are at high risk. Our study and most of the others were performed before these important preventive interventions entered clinical practice. The low numbers of participants in our study who were taking $\beta$-blockers and antihypertensive medications were not unusual in the 1980s and 1990s, but we cannot exclude underreporting of these data.

Finally, failure to reach statistical significance in some of our analyses and the wide confidence intervals indicate limited power of this study to detect differences among groups.

\section{Conclusions}

Men with incident type 2 diabetes without cardiovascular disease and men with a first acute cardiovascular event had similar mortality during long-term follow-up. The risk of cardiovascular death was at least 3 to 4 times greater in these 2 groups than among men with neither cardiovascular disease nor diabetes. Recent studies have reported a reduction in the rate of cardiovascular disease occurring in participants with diabetes, which may be attributable to more effective management and more aggressive use of preventive treatments. ${ }^{28,29}$ However, the burden of cardiovascular disease is likely increasing because of the continuing rise in the number of people newly diagnosed with or at high risk of diabetes. ${ }^{28}$ The implications of these findings underscore the importance of optimal management of this disease and its associated cardiovascular conditions, as well as the importance of pursuing research to prevent type 2 diabetes altogether.

This article has been peer reviewed.

Competing interests: None declared.

Contributors: All of the authors contributed to the study conception and design and to drafting and revising the article. All of the authors gave final approval of the version submitted for publication.

Funding: This study was supported in part by the Heart and Stroke Foundation of Canada and an unrestricted grant from Sanofi-Aventis and Merck Frosst Canada. The authors designed and coordinated the study and analyzed and discussed the results without any consultation with the supporting and sponsoring agencies.

\section{REFERENCES}

1. Zimmet P, Alberti KG, Shaw J. Global and societal implications of the diabetes epidemic. Nature 2001;414:782-7.

2. Kannel WB, McGee DL. Diabetes and cardiovascular disease: The Framingham Study. JAMA 1979;241:2035-8

3. Fox CS, D'Agostino RB Sr, Sullivan L, et al. The significant effect of diabetes duration on coronary heart disease mortality. The Framingham Heart Study. Diabetes Care 2004;27:704-8.

4. Butler WJ, Ostrander LD Jr, Carman WJ, et al. Mortality from coronary heart disease in the Tecumseh study. Long-term effect of diabetes mellitus, glucose tolerance and other risk factors. Am J Epidemiol 1985;121:541-7.

5. Pyörälä K, Laakso M, Uusitupa M. Diabetes and atherosclerosis: an epidemiologic view. Diabetes Metab Rev 1987;3:463-524.

6. Jarrett RJ, Shipley MJ. Type 2 (non-insulin-dependent) diabetes mellitus and cardiovascular disease - putative association via common antecedents; further evidence from the Whitehall Study. Diabetologia 1988;31:737-40.

7. Barrett-Connor EL, Cohn BA, Wingard DL, et al. Why is diabetes mellitus a stronger risk factor for fatal ischemic heart disease in women than in men? The Rancho Bernardo Study. JAMA 1991;265:627-31.

8. Lowe LP, Liu K, Greenland P, et al. Diabetes, asymptomatic hyperglycemia and 22-year mortality in black and white men. The Chicago Heart Association Detection Project in Industry Study. Diabetes Care 1997;20:163-9.

9. Adlerberth AM, Rosengren A, Wilhelmsen L. Diabetes and long-term risk of mortality from coronary and other causes in middle-aged Swedish men. A general population study. Diabetes Care 1998;21:539-45.

10. Vilbergsson S, Sigurdsson G, Sigvaldason H, et al. Coronary heart disease mortality amongst non-insulin-dependent diabetic subjects in Iceland: the independent effect of diabetes. The Reykjavik Study 17-year follow-up. J Intern Med 1998;244:309-16.

11. Rodriguez BL, Sharp DS, Lau N, et al. Glucose intolerance and 23-year risk of coronary heart disease and total mortality. The Honolulu Heart Program. Diabetes Care 1999;22:1262-5.

12. Haffner SM, Lehto S, Rönnemaa T, et al. Mortality from coronary heart disease in subjects with type 2 diabetes and in nondiabetic subjects with and without prior myocardial infarction. N Engl J Med 1998;339:229-34.

13. Booth GL, Kapral MK, Fung K, et al. Relation between age and cardiovascular disease in men and women with diabetes compared with nondiabetic people: a population-based retrospective cohort study. Lancet 2006;368:29-36.

14. Whiteley L, Hole D, Padmanabhan S, et al. Should diabetes be considered a coronary heart disease risk equivalent? Results from 25 years of follow-up in the Renfrew and Paisley Survey. Diabetes Care 2005;28:1588-93.

15. Hu FB, Stampfer MJ, Solomon CG, et al. The impact of diabetes mellitus on mortality from all-causes and coronary heart disease in women. Arch Intern Med 2001;161:1717-23. 
16. Natarajan S, Liao Y, Cao G, et al. Sex differences in risk for coronary heart disease mortality associated with diabetes and established coronary heart disease. Arch Intern Med 2003; 163:1735-40.

17. Becker A, Bos G, de Vegt F, et al. Cardiovascular events in type 2 diabetes: comparison with nondiabetic individuals without and with prior cardiovascular disease. Eur Heart J 2003;24:1406-13.

18. Lotufo PA, Gaziano JM, Chae CU, et al. Diabetes and all-cause and coronary heart disease mortality among US male physicians. Arch Intern Med 2001;161:242-7.

19. Cho E, Rimm EB, Stampfer MJ, et al. The impact of diabetes mellitus and prior myocardial infarction on mortality from all causes and from coronary heart disease in men. J Am Coll Cardiol 2002;40:954-60.

20. Vaccaro O, Eberly LE, Neaton JD, et al. Impact of diabetes and previous myocardial infarction on long-term survival: 25 -year mortality follow-up of primary screenees of the Multiple Risk Factor Intervention Trial. Arch Intern Med 2004; $164: 1438-43$.

21. Eberly LE, Cohen JD, Prineas R, et al. Impact of incident diabetes and incident nonfatal cardiovascular disease on 18-year mortality. Diabetes Care 2003;26: 848-54.

22. Evans JM, Wang J, Morris AD. Comparison of cardiovascular risk between patients with type 2 diabetes and those who had had a myocardial infarction: cross sectional and cohort studies. BMJ 2002;324:939-42.

23. Lee CD, Folsom AR, Pankow JS, et al. Cardiovascular events in diabetic and nondiabetic adults with and without history of myocardial infarction. Circulation 2004;109:855-60.
24. Robitaille NM, Christen A, Dagenais GR, et al. Prévalence des facteurs de risque de la maladie coronarienne dans la région de Québec. Clin Invest Med 1979;2: 13-16.

25. Dagenais GR, Robitaille NM, Lupien PJ, et al. First coronary heart disease event rates in relation to major risk factors: Québec Cardiovascular Study. Can J Cardiol 1990;6:274-80.

26. Colditz GA, Martin P, Stampfer NJ. Validation of questionnaire information on risk factors and disease outcome in a prospective cohort study of women. Am J Epidemiol 1986;123:894-900.

27. Brunner EJ, Shiply MJ, Witte DR, et al. Relation between blood glucose and coronary mortality over 33 years in the Whitehall Study. Diabetes Care 2006;29:26-31.

28. Booth GL, Fung K, Kapral MK, et al. Recent trends in cardiovascular complications among men and women with and without diabetes. Diabetes Care 2006;29:32-7

29. Fox CS, Pencina MJ, Meigs JB, et al. Trends in the incidence of type 2 diabetes mellitus from 1970s to the 1990s. The Framingham Heart Study. Circulation 2006;113:2914-8.

Correspondence to: Dr. Gilles R. Dagenais, Institut universitaire de cardiologie et pneumologie, 2725, chemin Ste-Foy, Québec QC G1V4G5; fax418656-4683; gilles.dagenais@crhl.ulaval.ca

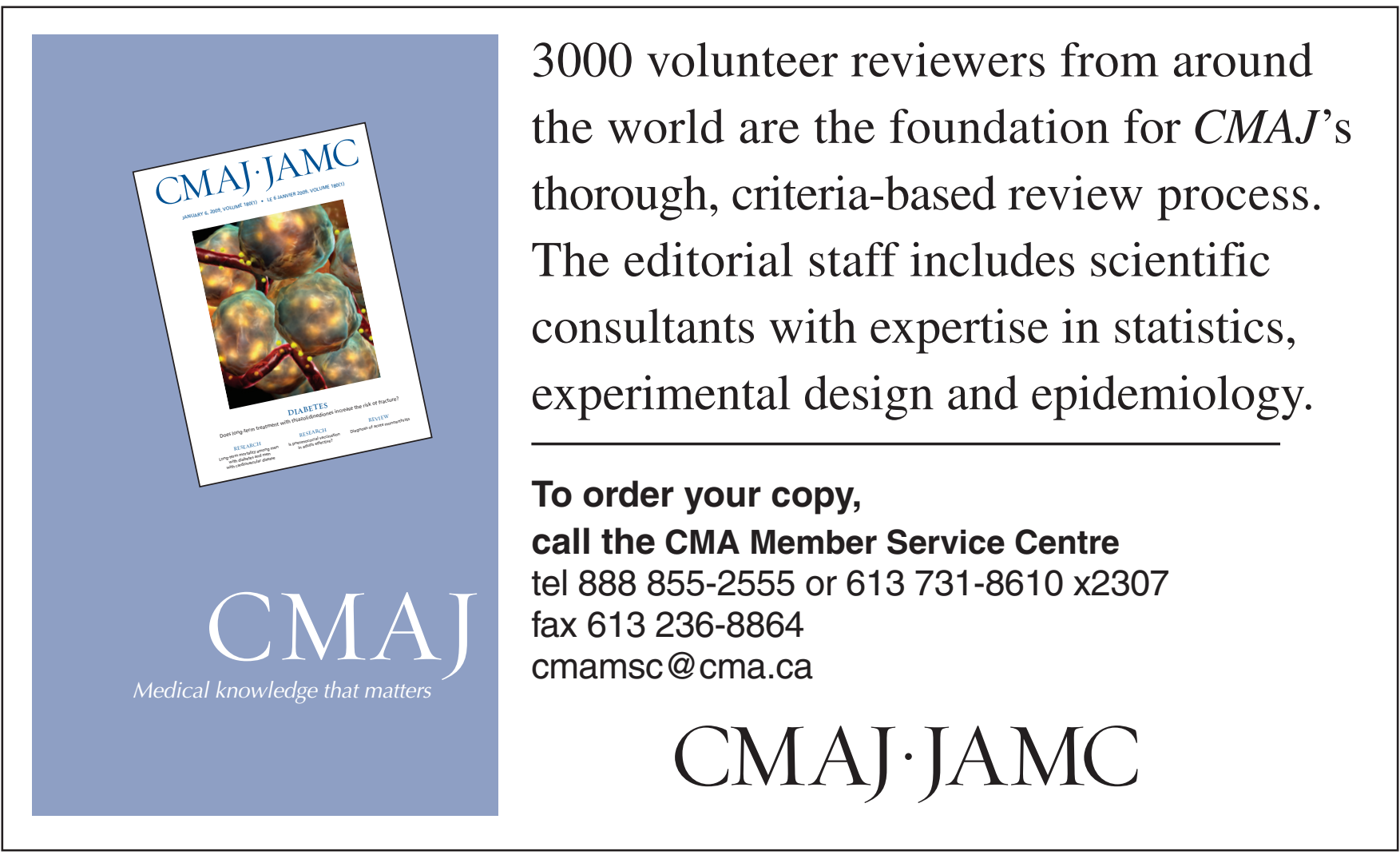

